\title{
Wavelet Energy based Neural Fuzzy Model for Automatic Motor Imagery Classification
}

\author{
Girisha Garg \\ ICE Department \\ NSIT, New Delhi
}

\author{
Shruti Suri \\ ICE Department \\ NSIT, New Delhi
}

\author{
Rachit Garg \\ ICE Department \\ NSIT, New Delhi
}

\author{
Vijander Singh \\ ICE Department \\ NSIT, New Delhi
}

\begin{abstract}
Brain-computer interface (BCI) is a communication system by which a person can send messages without any use of peripheral nerves and muscles. BCI systems might help to restore abilities to patients who have lost sensory or motor function because of the damaged region, such as amyotrophic lateral sclerosis (ALS), spinal cord injury, brainstem stroke, or quadriplegic patients. Brain computer interfacing can be effectively implemented by analyzing EEG signals generated in the brain. This paper presents a method for accurately classifying EEG signals generated by imagery left and right hand movements. Firstly, wavelet transform and energy of the decomposed signal is used to obtain the final feature vector matrix. Secondly, the feature data is classified using ANFIS. . The Mutual Information value calculated is 1.2942 bit. The classification accuracy achieved $93.5 \%$ in the course of testing on the data from subject. Support Vector Machine is also used to compare the performance with ANFIS.
\end{abstract}

Keywords: ANFIS, EEG signals, SVM, wavelet transform, motor imagery

\section{INTRODUCTION}

Recent times have seen a significant increase in the number of people showing interest in developing new brain computer interfacing methods. BCI technology can be extremely useful in assisting, augmenting or repairing human cognitive or sensorymotor functions. The Electroencephalogram (EEG) is used to measure the brain signals pertaining to various activities like imagining hand movements etc. The EEG recognition procedure mainly involves feature extraction and classification. Numerous methods have been used to extract feature vectors from the EEG signals. Wavelet transform is used as an effective means of feature vector extraction. Basis functions of the wavelet transform are small waves located in different times. They are obtained using scaling and translation of a scaling function and wavelet function. Therefore the wavelet transform is localised in both time and frequency. In addition the wavelet transform also provides a multi resolution system, which is useful in several applications like image communications and image database. The extracted feature vectors are then classified using machine learning techniques like neural networks, linear discriminant analysis, support vector machines (SVM), common spatial pattern, etc. Feature extraction and pattern classification of EEG is a difficult problem during the research of BCI. Several researches in this field have been put forth. Huaiyu $\mathrm{Xu}^{*}$ et. al [1] have proposed a technique which combines wavelet coefficient feature represented time domain with the power spectral density represented frequency domain to form a feature vector which is then used for classification purpose. Xiaoyan Qiao and Yanjing Wang, Douzhe Li, Lifeng Tian[2] used svm classifiers and misclassification rate. Guodong Shil Zhenghua $\mathrm{Ma}$ [3] et. al used discrete wavelet transform along with fisher linear discriminant analysis. Various such methods $[4,5,6]$ have been used with acceptable accuracy, but at the same time unexpected limitations have also existed, such as depending closely on many unrelated factors, needing to process a large amount of data, etc. In this paper, a new method for the recognition of electroencephalography (EEG) has been proposed, which includes the wavelet transform, Adaptive-Network-Based Fuzzy Inference Systems (ANFIS) and Support Vector Machine (SVM). Classification results obtained by ANFIS and SVM are effective.

\section{METHODOLOGY}

The main objective of this paper is to test the performance of the proposed system for the recognition of EEG signals in the motor imagery domain. The system's architecture for such an automated system is shown in Fig.1
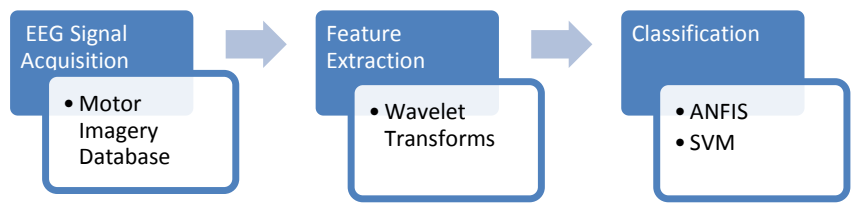

Figure 1: System Architecture

\subsection{Wavelet Transform}

Earlier FFT (Fast Fourier Transform) was widely used technique to calculate the power spectral density of the frequency bands. But Fourier transforms can only localize in frequency and do not work well in real time. A relatively new nonparametric method 
based on time frequency analysis is Wavelet Transforms (WT) which can overcome the drawbacks, such as spectral leakage and cross modulation suffered by the classical Fourier techniques, and their evolutions like the Short Time Fourier Transform (STFT). The WT allows obtaining better temporal resolution for higher frequency components and poorer temporal resolution (but better frequency resolution) for the lower frequency components i.e., multi resolution analysis which is highly required for the non stationary signals like EEG [40, 41]. Wavelet transform is a method of multi-resolution time frequency analysis, which can decompose the mixed signals which consist of different frequencies into different frequency band .WT decomposes a signal into small waves with energy concentrated in time called wavelets. These wavelets are the scaled and shifted copies of the main pattern, so-called the "mother wavelet" The mother wavelet function is defined by equation (1), where $b$ is translation parameter and, a as scale parameter.

$$
\psi_{a, b}(t)=\frac{1}{\sqrt{a}} \psi\left(\frac{t-b}{a}\right)
$$

DWT analyses the signal using Multi Resolution analysis (MRA) by decomposing the signal into approximations and detail information by employing two functions: scaling and wavelet function as shown in equation 1 The approximation coefficient is subsequently divided into new approximation and detailed coefficients which are shown in equation (2) given below. This process is carried out iteratively producing a set of approximation coefficients (CA) and detailed coefficients (CD) at different levels $(\mathrm{N})$ of decomposition.

For a given signal $\mathrm{x}(\mathrm{t})$ the DWT decomposition can be represented by equation (2).

$$
\begin{gathered}
x(t)=\sum_{k=-\infty}^{k=+\infty} C_{N, k} \phi\left(2^{-N} t-k\right) \\
+\quad \sum_{j=1}^{N} \sum_{k=-\infty}^{k=+\infty} d_{j, k} 2^{-j / 2} \psi\left(2^{-j} t-k\right)
\end{gathered}
$$

Where $\mathrm{CN}, \mathrm{k}$ represents approximation coefficients at level $\mathrm{N}$, while $\mathrm{dj}, \mathrm{k}(\mathrm{j}=1$ to $\mathrm{N})$ represents detailed coefficients or wavelet coefficients at level $\mathrm{j} . \psi(\mathrm{t})$ is the wavelet function, while $\varphi(t)$ is a companion function, named as scaling function. Wavelet transform is used for EEG feature extraction in our research.

\subsection{Wavelet Energy}

If the scaling functions and wavelets form an orthogonal basis, Parseval's theorem relates the energy of the signal $x(t)$ to the energy in each of the components and their wavelet coefficients. The energy of the detailed signal at each resolution level, $j$ is given by:

$$
E_{j}=\sum_{k}\left|d_{j, k}\right|^{2} j=1 \text { toN } \quad \ldots 3
$$

The wavelet energy can be used to extract only the useful information from the signal about the process under study. Wavelet Energy gives the information about energy associated with the frequency bands and can detect the degree of similarity between segments of a signal. . Thus this method accurately detects and characterizes the specific phenomenon related to the different frequency bands of the EEG signal. It gains an advantage over DWT based feature extraction in terms of speed, computation efficiency and classification rate.

\subsection{Support Vector Machine Classifier}

A support vector machine (SVM) is a new machine learning method that analyzes data and recognize patterns, used for classification and regression analysis. SVM has been used in many works related to the classification of EEG signals. Application of SVM for sleep classification and epileptic seizure detection is presented in [37] and [23] respectively. SVM developed base on VC dimension theory and structural risk minimization principle in the statistical learning theory. The standard SVM takes a set of input data and predicts, for each given input, which of two possible classes the input is a member of, which makes the SVM a non-probabilisticbinarylinear classifier. Given a set of training examples, each marked as belonging to one of two categories, an SVM training algorithm builds a model that assigns new examples into one category or the other. A support vector machine constructs a hyperplane or set of hyperplanes in a high- or infinite- dimensional space, which is then used for classification, regression, or other tasks.

Suppose we are given a set $\mathrm{S}$ of labeled training points (y1, $\mathrm{x} 1) \ldots . . . .(\mathrm{yn}, \mathrm{xn})$. Each training point xi belongs to either of two classes and is given a label yi $\in\{-1,1\}$ for $i=1$ to $n$. We wish to find the hyperplane

$w \cdot \boldsymbol{x}+\boldsymbol{b}=\mathbf{0}$

defined by the pair $(\omega, b)$, such that the inequality

$y_{i}\left(w \cdot x_{i}+b\right) \geq 1-\xi_{i}$

holds, where $\xi_{\mathrm{i}} \geq 0$ is the slack variable for the data points that cannot be made to fit in the optimal hyperplane. The optimal hyperplane problem is then regarded as the solution to the problem

Minimize

$\frac{1}{2} w \cdot w+C \sum_{i=1}^{n} \xi_{i}$

Subject to:

$y_{i}\left(w \cdot x_{i}+b\right) \geq 1-\xi_{i}$ ... 7

$\xi_{i} \geq 0$,

where $\mathrm{C}$ is a constant. The parameter $\mathrm{C}$ can be regarded as a regularization parameter. This is the only free parameter in the SVMs formulation. Tuning this parameter can make balance between margin maximization and classification violation. Searching the optimal hyperplane is a quadratic programming (QP) problem, that can be solved by constructing a Lagrangian function and transformed into its dual Maximize

$W(\alpha)=\sum_{i=1}^{l} \alpha_{i} y_{i}-\frac{1}{2} \sum_{i=1}^{n} \sum_{j=1}^{n} \alpha_{i} \alpha_{j} y_{i} y_{j} x_{i} x_{j}$ ... 9 
Subject to

$\sum_{i=1}^{n} \alpha_{i} y_{i}=0$

$0 \leq \alpha_{i} \leq C$

Where $\boldsymbol{\alpha}=\left(\alpha_{1}, \ldots, \alpha_{n}\right)$ is the vector of non-negative Lagrange multipliers associated with the constraints. According to the Kuhn-Tucker theorem, the solution $\alpha_{\mathrm{i}}$ of problem (9) satisfies the equality

$\alpha_{i}\left(y_{i}\left(w \cdot x_{i}+b\right)-1+\xi_{i}\right)=0 \quad \ldots 12$

$\left(C-\alpha_{i}\right) \xi_{i}=0 \quad$.

To construct the optical hyperplane, it follows that = $\sum_{i=1}^{l} \alpha_{i} x_{i} y_{i}$, thus the decision function can be written as:

$f(x)=\operatorname{sign}\left(\sum_{i=1}^{l} \alpha_{i} x_{i} y_{i} \cdot x+b\right)$

where

$\operatorname{sign}(x)=\left\{\begin{array}{c}1, x>0 \\ 0, x=0 \\ -1, x<0\end{array}\right.$

When the decision function is not a linear function of the data, the data is firstly mapped to a high-dimensional feature space $\mathrm{F}$, in which the data is linearly separable. For the purpose, $x_{i} . x$ in the $f(x)$ will be replaced by the kernel function $K\left(x_{i}, x\right)=$ $\phi\left(x_{i}\right) . \phi(x)$, then the decision function is reformulated as:

$f(x)=\operatorname{sign}\left(\sum_{i=1}^{l} \alpha_{i} y_{i} K\left(x_{i} \cdot x\right)+b\right) \quad \ldots 16$

An example of polynomial kernel is as follows:

$$
K(x, y)=\left[\left(x^{T} \cdot y\right)+1\right]^{p}
$$

Where $\mathrm{p}$ is the degree of the polynomial.

SVM has been used in several medical based applications. It provides a robust method for classification by minimizing the over fitting problems by adopting the principle of structural risk minimization. A more detailed theory on SVM can be found in [42].

\subsection{Adaptive Neuro-Fuzzy Inference System}

This is the major training routine for Sugeno-type fuzzy inference systems. Anfis uses a hybrid learning algorithm to identify parameters of Sugeno-type fuzzy inference systems. It applies a combination of the least-squares method and the backpropagation gradient descent method for training FIS membership function parameters to emulate a given training data set. ANFIS is an adaptive network. It is a network of nodes and directional links. It is called adaptive because some, or all, of the nodes have parameters which affect the output of the node. These networks are learning a relationship between inputs and outputs. Fig 2 shows the ANFIS architecture. The circular nodes represent nodes that are fixed whereas the square nodes are nodes that have parameters to be learnt.

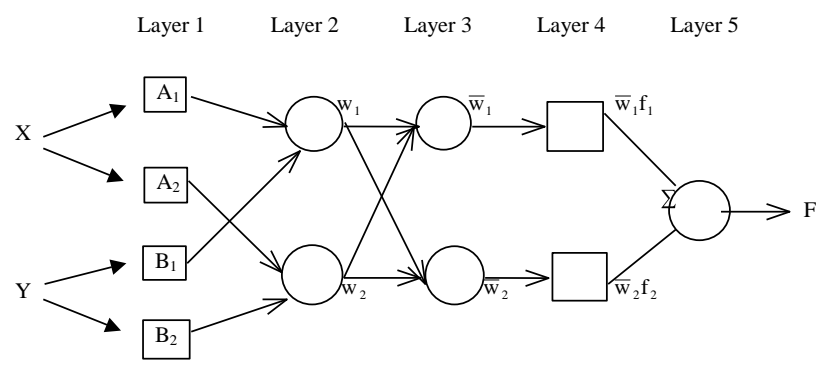

Figure 2:An ANFIS architecture for a two rule Sugeno system

A Two Rule Sugeno ANFIS has rules of the form:

$$
\begin{aligned}
& \text { If } x \text { is } A_{1} \text { and } y \text { is } B_{1} \text { THEN } f_{1}=p_{1} x+q_{1} y+r_{1} \\
& \text { If } x \text { is } A_{2} \text { and } y \text { is } B_{2} \text { THEN } f_{2}=p_{2} x+q_{2} y+r_{2}
\end{aligned}
$$

One possible ANFIS architecture to implement these two rules is shown in Fig. 6. Note that a circle indicates a fixed node whereas a square indicates an adaptive node (the parameters are changed during training).

Layer 1: All the nodes in this layer are adaptive nodes. $\mathrm{i}$ is the degree of the membership of the input to the fuzzy membership function (MF) represented by the node. The output of each node is given by

$$
\begin{aligned}
& O_{1, i}=\mu_{A_{i}}(x) \quad \text { for } i=1,2 \\
& O_{1, i}=\mu_{B_{i-2}}(y) \quad \text { for } i=3,4
\end{aligned}
$$

where, $\mathrm{O} 1, \mathrm{i}(\mathrm{x})$ is essentially the membership grade for $\mathrm{x}$ and $\mathrm{y}$. $\mathrm{Ai}$ and $\mathrm{Bi}$ can be any appropriate fuzzy sets in parameter form. For example, if bell MF is used then

$$
\mu_{A}(x)=\frac{1}{1+\left|\frac{x-c_{i}}{a_{i}}\right|^{2 b_{i}}}
$$

Where ai,, bi and ci are the parameters for the MF.

Layer 2: The nodes in this layer are fixed (not adaptive). They play the role of a simple multiplier. The outputs of these nodes are given by

$$
O_{2, i}=w_{i}=\mu_{A_{i}}(x) \mu_{B_{i}}(y), \quad i=1,2
$$

Layer 3: Nodes in this layer are also fixed. They perform a normalization of the firing strength from previous layer. The output of each node in this layer is given by 
$O_{3, i}=\bar{w}_{i}=\frac{w_{i}}{w_{1}+w_{2}}$

Layer 4: All the nodes in this layer are adaptive nodes. The output of each node is simply the product of the normalized firing strength and a first-order polynomial

$O_{4, i}=\overline{w_{i}} f_{i}=\overline{w_{i}}\left(p_{i} x+q_{i} y+r_{i}\right)$

Where pi, qi and ri are design parameters.

Layer 5: This is a single node layer which performs the function of a simple summer. The output of this single node is given by

$O_{5, i}=\sum_{i} \overline{w_{i}} f_{i}=\frac{\sum_{i} w_{i} f_{i}}{\sum_{i} w_{i}}$ ... 24

The ANFIS architecture is not unique. Some layers can be combined and still produce the same output. In this ANFIS architecture, there are two adaptive layers (1 and 4). Layer 1 has three modifiable parameters (ai,, bi and ci) pertaining to the input MFs. These parameters are called premise parameters. Layer 4 has also three modifiable parameters (pi, qi and ri) pertaining to the first-order polynomial. These parameters are called consequent parameters.

Learning algorithm: The learning algorithm is used to update the parameters associated with the membership function. The updating of these parameters is facilitated by a gradient vector which provides a measure of how well the fuzzy inference system is modelling the input/output data for a given set of parameters. Further the parameters are adjusted using any of approach mentioned below to some error measure.In this paper the hybrid algorithm is used for its non complexity and high efficiency in training.A hybrid algorithm adjusts the consequent parameter pi, qi and ri in a forward pass and the premise parameters ai,, bi and ci in a backward pass. In the forward pass the network inputs propagate forward until layer 4, where the consequent parameters are identified by the least-squares method. Once the optimal consequent parameters are found, the backward pass starts immediately. In the backward pass, the error signals propagate backwards and the gradient descent method is used to update the premise parameters corresponding to the fuzzy sets in the input domain. The output of the ANFIS is calculated by employing the consequent parameters found in the forward pass. The output error is used to adapt the premise parameters by means of a standard back propagation algorithm.

\section{WORK DONE}

\subsection{Data Description}

Data set was provided by Department of Medical Informatics, Institute for Biomedical Engineering, University of Technology Graz [7].This dataset was recorded from a normal subject (female, 25y) during a feedback session. The subject sat in a relaxing chair with armrests. The task was to control a feedback bar by means of imagery left or right hand movements. The order of left and right cues was random.

The experiment consists of 7 runs with 40 trials each. All runs were conducted on the same day with several minutes break in between. Given are 280 trials of 9 s length. The first 2 s was quite, at $\mathrm{t}=2 \mathrm{~s}$ an acoustic stimulus indicates the beginning of the trial, the trigger channel (\#4) went from low to high, and a cross "+" was displayed for $1 \mathrm{~s}$; then at $\mathrm{t}=3 \mathrm{~s}$, an arrow (left or right) was displayed as cue. At the same time thesubject was asked to move a bar into the direction of a the cue. The feedback was based on AAR parameters of channel \#1 (C3) and \#3 (C4), the AAR parameters were combined with a discriminant analysis into one output parameter. (similar to $[1,2])$. The recording was made using a G.tec amplifier and a $\mathrm{Ag} / \mathrm{AgCl}$ electrodes. Three bipolar EEG channels (anterior '+', posterior '-') were measured over $\mathrm{C} 3, \mathrm{Cz}$ and $\mathrm{C} 4$. The EEG was sampled with $128 \mathrm{~Hz}$, it was filtered between 0.5 and $30 \mathrm{~Hz}$.

The trials for training and testing were randomly selected. This should prevent any systematic effect due to the feedback.
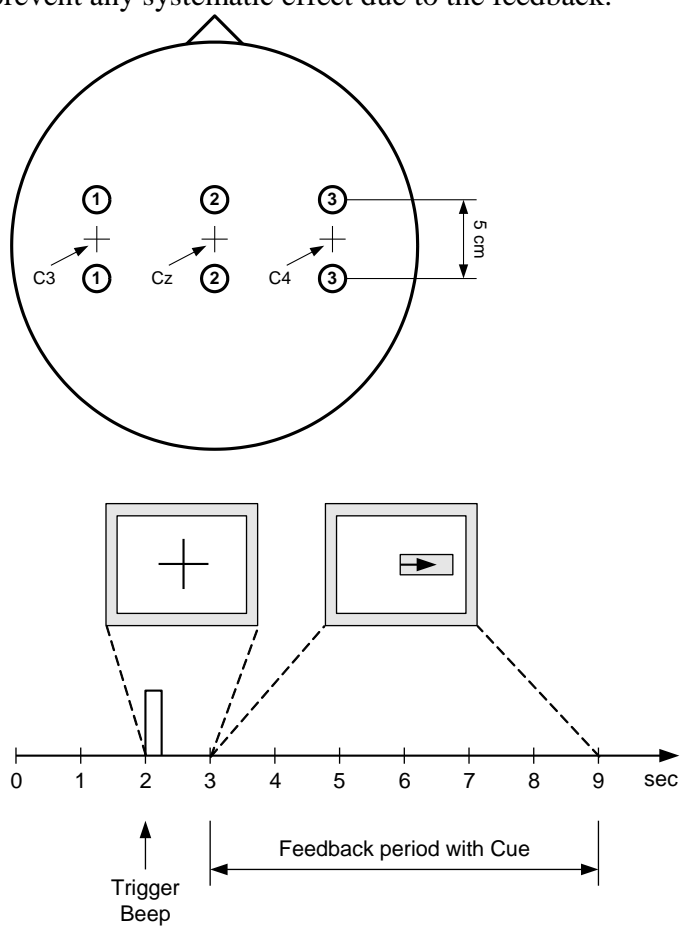

Figure 3: Electrode positions (left) and timing scheme (right)

\subsection{Feature Extraction and Classification}

The EEG signals in the database are segregated into 2 set: 1. Training Set

2. Test Set

The training set comprises of a 100 samples and the test set consists of 40 samples. The training samples are further divided into the left hand and right hand samples. The right and the left hand samples are then used in the following stages of operation: 


\subsubsection{Feature Extraction}

The training set comprises of a 100 samples and the test set consists of 40 samples. The training samples are further divided into the left hand and right hand samples. Wavelet transform is used for EEG feature extraction in our research. It decomposes the signal into different frequency bands shown in figure. 4 .

\section{Decomposition:}

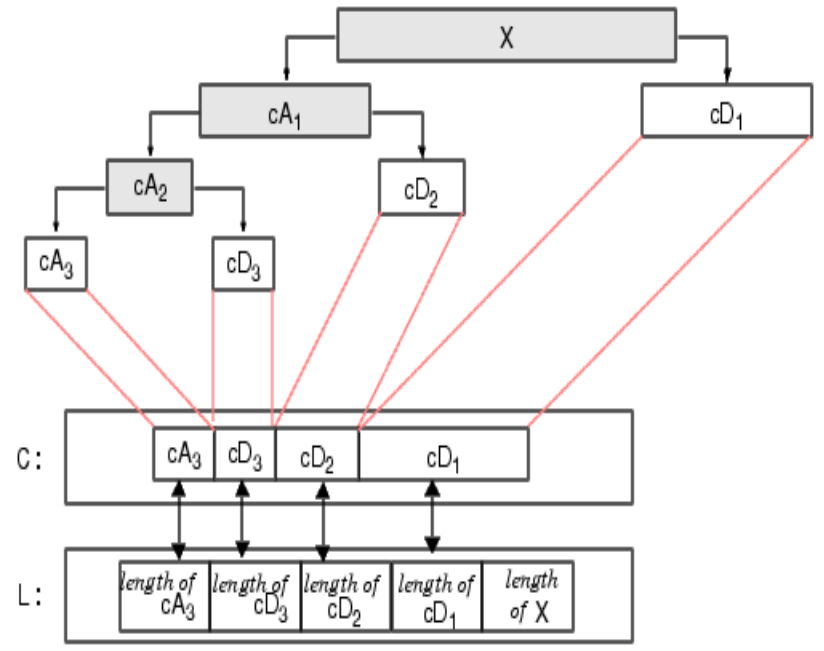

Figure 4: Wavelet decomposition for level 3

Thus, keeping in mind the frequency of the EEG samples, wavelet transform with varying levels of decomposition is performed to optimize the feature extraction. Finally, the level of decomposition and the type of wavelet was chosen to be 4 and $\mathrm{db} 3$ respectively. The signals so obtained are decomposed using $\mathrm{db} 3$ as the mother wavelet and the decomposition level as 4.

Once the wavelet energy features are extracted from all three EEG channels i.e. C3, C4 and CZ, a study is made to find out the most effective features which can show a clear distinction between the two imagery functions. Because channel $\mathrm{CZ}$ showed its independence of the motor imagery, the signals recorded from the $\mathrm{C} 3$ and $\mathrm{C} 4$ electrodes were chosen for the research and used for the EEG signal recognition. The wavelet energy of the decomposed signals of channels C3 and C4 was calculated.The difference between the energy values of the two electrodes is plotted as shown in Figure 5.

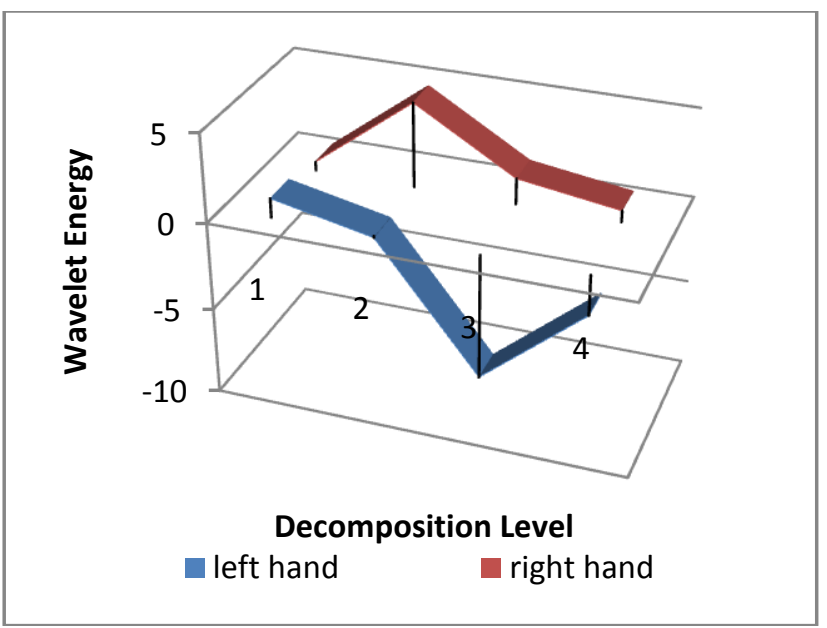

Figure 5. The feature signals of the left and right hands imagery movement

From the figure, it is concluded that the difference value of the energies well reflect the distinction of imaginary left and right hand movements. Therefore, the two energies are used as the final feature vector for classification.

Final Feature Vector $=\operatorname{Energy}(\mathrm{C} 3)-\operatorname{Energy}(\mathrm{C} 4) \quad \ldots 25$

The wavelet energy features extracted form the EEG signals of the two stages are then used for the classification purposes. This is done using the ANFIS and SVM classifiers.

\subsubsection{Feature Classification}

After the completion of the feature extraction stage, the extracted features are classified in order to show the effectiveness of the BCI application. Several classification techniques have been used till date.In this paper we compare two different methods of feature classification namely (1) Support Vector Machine (SVM) and (2) Adaptive Neuro Fuzzy Inference System (ANFIS). For SVM, 1 and -1 are used as the two class labels whereas for ANFIS 1 and 2 are used to represent two different sets of data. It is seen that the ANFIS classifier is able to give better results as compared to the SVM classifier. The accuracy achieved with the SVM classifier is $72 \%$ whereas the accuracy achieved with the ANFIS classifier is approximately $93 \%$. Therefore the designing of ANFIS classifier is briefly described.

For the ANFIS classifier, the fuzzy rule architecture is designed by using two membership functions. The Generalized bellshaped function is used as the input function and linear function is used as the output function. To train the parameters associated with the membership functions of the fuzzy system the hybrid algorithm is used as the learning process, which is a combination of least square and back propagation method.The whole procedure explained above is summarized in Fig. 6 shown below. 


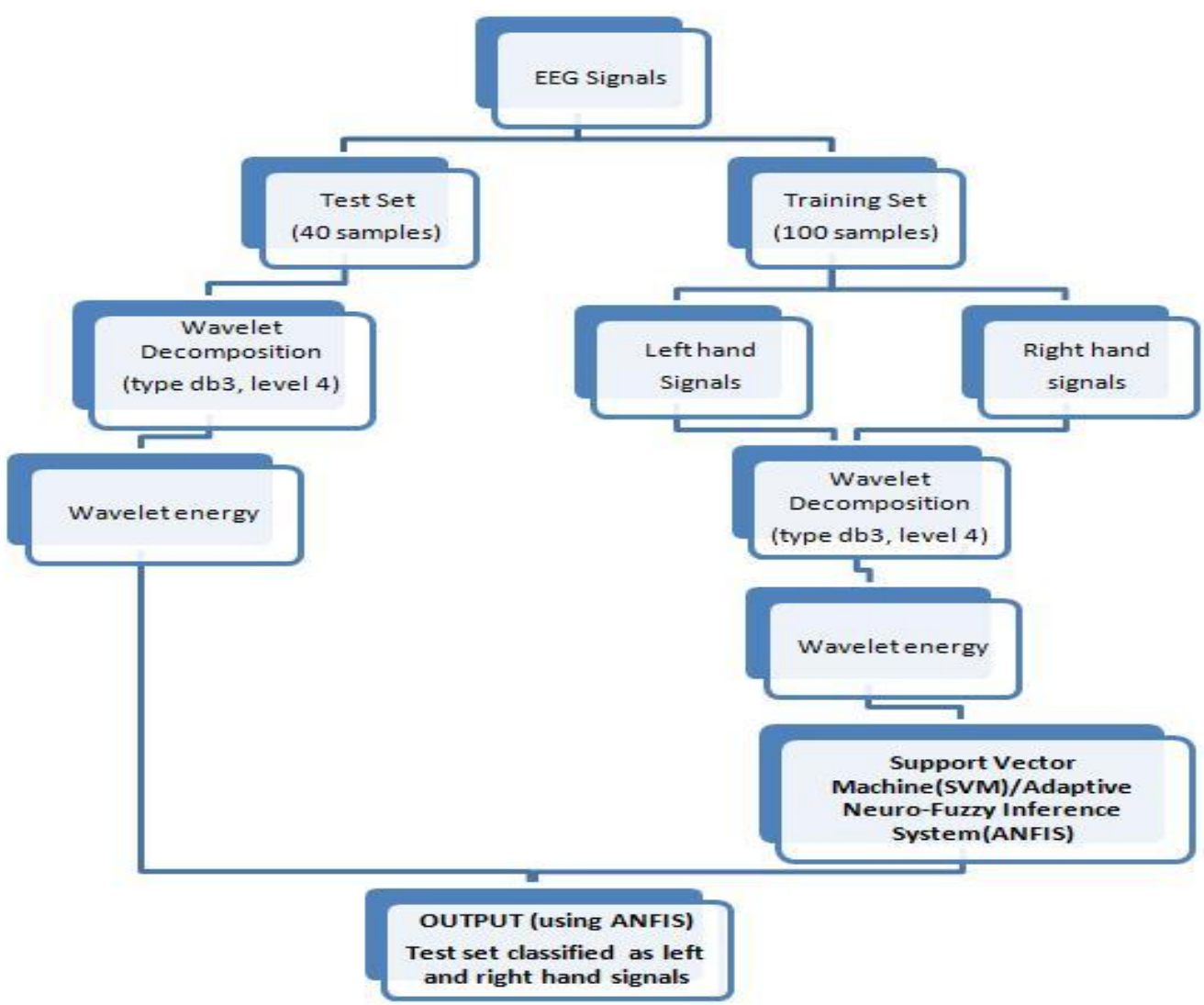

Figure 6: Steps involved in feature extraction and classification

\section{RESULTS AND DISCUSSIONS}

The system described by Fig. 6 is used to classify the motor imagery EEG signals as left or right. The complete training set is identified correctly by both the classifiers, SVM and ANFIS. The accuracy obtained for ANFIS is $93.5 \%$, which is higher than that obtained for SVM $(72 \%)$.

In this paper an automated system, using wavelet energy and ANFIS, is provided for the feature vector extraction and classification of the EEG signal as well as a comparative analysis between SVM and ANFIS is presented. For feature extraction the db2 mother wavelet is used. The selection of decomposition level depends on the spectral content of the EEG signal. The decomposition level is chosen as 4.For values of the decomposition level more than 4 it is observed that the accuracy of the system starts decreasing.

In order to verify the results obtained, mutual information (MI) obtained from classifying results is used as the criterion to compare the performances of different methods.Greater the value of the MI better are the classification results by a classifier. In this paper, the classifying results obtained by the ANFIS based on the proposed feature set are extensively compared with the ones achieved by the BCI-competition winning methods on the same data set in terms of the criteria of MI. The transmitted information rate of BCI data is quantified by Shannon'scommunication theoryand obtained from the following formula of SNR and MI [9].

$$
\begin{aligned}
& S N R_{t}=\frac{2 * \operatorname{var}_{i \in L, R} D_{t}^{(i)}}{\operatorname{var}_{i \in L} D_{t}^{(i)}+\operatorname{var}_{i \in L} D_{t}^{(i)}}-1 \\
& M I_{t}=0.5^{*} \log _{2}\left(1+S N R_{t}\right)
\end{aligned}
$$

Where, $S N R_{i}$ is the SNR of BCI system at t time point; $\operatorname{var}\{$.$\} is$ the variance of all experiments; $i$ is the time of experiment; $D_{t}^{(i)}$ is the discrimination distance of experiment $i$ at $t$ time point, reflecting classification reliability; $\{L\}$ and $\{R\}$ represent imagination of left and hand movements respectively; $M I_{t}$ is the mutual information between input and output of BCI system at $t$ time point. The amount of mutual information can be used for evaluation of EEG signal feature extraction and classification method.Table 1 ranks the performances of the ANFIS method, the BCI Competition winning methods and the WEP-SVM method in [2] in terms of the MI. 
TABLE 1. Ranking Order of The Proposed Method and theBCICompetition Winning MethodsTogether With the Methods in [2] on The Graz Dataset ofBCI Competition inTermsofTheMI

\begin{tabular}{|l|c|}
\hline \multicolumn{1}{|c|}{ METHODS } & MAXIMAL MI(BIT) \\
\hline $\begin{array}{l}\text { ADAPTIVE NEURO-FUZZY } \\
\text { INFERENCE SYSTEM } \\
\text { (PROPOSEDMETHOD) }\end{array}$ & 1.2942 \\
\hline SVM WITH WEP[2] & 0.65 \\
\hline BCI_COMP 1 ${ }^{\text {ST } \quad \text { WINNER }}$ & 0.61 \\
\hline BCI_COMP $2^{\text {ND }}$ WINNER & 0.46 \\
\hline BCI_COMP $3^{\text {RD }}$ WINNER & 0.45 \\
\hline
\end{tabular}

\section{CONCLUSION}

EEG is used as a valuable tool in the diagnosis, monitoring and managing of the various neurological disorders. The segregation and the classification of the EEG signals taken from various brain regions is an important technique to implement brain computer interfacing. The research presents the use of ANFIS as a new promising algorithm for the analysis of the EEG data. It is observed that the proposed technique is a robust, fast and efficient technique for EEG signal classification. Initially the EEG signals are decomposed to a particular decomposition level. The energy of the decomposed signal is the calculated and the feature vector matrix is obtained using Wavelet Transform. Finally the extracted features are then classified using ANFIS. The results presented here also draw a comparison between two techniques of EEG signal classification SVM and ANFIS. The research shows that both the ANFISachieves a high accuracy of 93.5\%. Mutual information (MI), an evaluation criterion was utilized to evaluate the performance of the classifier. The value of the MI is found to be 1.2942. Thus, the method introduced in this paper is a promising algorithm for the analysis of EEG data, deserving to be carefully validated and studied for its own sake.

\section{REFERENCES}

[1] Xu H., Lou J., Su R., Zhang E.,2009 "Feature extraction and classification of EEG for imaging left-right hands movement," iccsit,2nd IEEE International Conference on Computer Science and Information Technology.

[2] Qiao X., Wang Y., Li D., and Tian L.,2010 "Feature extraction and classifier evaluation of EEG for imaginary hand movements", in Proc. ICNC.

[3] Zou L., Guodong X.W., Ma S.Z.,2010 "EEG Feature extraction and pattern classification based on Motor Imagery in Brain-computer Interface", ICCI, 9th IEEE International Conference.
[4] Hu J. F., Bao X. C., and Mu Z.D.,2008 "Classification of Motor Imagery EEG Based on Phase Synchronization," Microelectronics and Computer.

[5] $\mathrm{Hu}$ J. F., Mu Z.D., and Xiao D.,2008 "Classification of motor imagery EEG signals based on energy entropy," Computer Engineering and Applications.

[6] Ramoser H., Muller-Gerking J., and Pfurtscheller G., "Optimal spatial filtering of single trial EEG during imagined hand movement", IEEE Trans. Rehabil. Eng.,

[7] Institute for Biomedical Engineering, Graz University of Technology,http://www.bbci.de/competition/ii/.

[8] Zhang L., Liu G., and Wu Y.,2010“Wavelet and Common Spatial Pattern for EEG Signal Feature Extraction and Classification," 2010 International Conference on Computer, Mechatronics, Control and Electronic Engineering (CMCE)

[9] Schlögl A., Kronegg Julien,HugginsE. Jane,Mason G. Steve, 2007 Evaluation Criteria for BCI Research. In Toward Brain-computer Interfacing.

[10] Sanej, Saeid and Chambers, J. A. EEG Signal PRocessing. WILEY, 2007.

[11] Ungureanu, M., Bigan, C. and Strungaru, R. 2, 2004, Independent Component Analysis Applied in Biomedical Signal Processing Measurement Science Review, Vol. 4, pp. 1-8.

[12] Tutorials, SVM. www.svms.org/tutorials/. www.svms.org. [Online].

[13] Srinivasan, V., Eswaran, C. and Sriram, N. 3, 2007, Approximate Entropy-Based Epileptic EEG Detection Using Artificial Neural Networks. Information Technology in Biomedicine, Vol. 11, pp. 288-295.

[14] G. Garg, V. Singh, J. R. P Gupta, A. P. Mittal, S. Chandra, "Computer Assisted Automatic Sleep Scoring System Using Relative Wavelet Energy Based Neuro Fuzzy Model" WSEAS Transactions on Biology and Biomedicine, Vol. 8, 2011.

[15] G. Garg, V. Singh, J.R.P Gupta A.P. Mittal, "Relative Wavelet Energy as a New Feature Extractor For Sleep Classification using EEG Signals" Accepted for publication in Journal of Biomedical Signal Processing. Jan- June Issue, 2011.

[16] Krausz G, Ortner R, Opisso, "Accuracy of a Brain Computer Interface (P300 Spelling Device) used by People with Motor Impairments" US National Library of MedicineNational Institutes of Health, 2011.

[17] Faradji F, Ward RK, Birch GE., "Toward development of a two-state brain-computer interface based on mental tasks" US National Library of MedicineNational Institutes of Health, 2011. 